\title{
无碰撞磁场重联中的电子密度空穴和 $\boldsymbol{B}_{\boldsymbol{y}}$ 的四极型分布
}

黄灿 $^{(12)}$ ，王荣生 ${ }^{(1)}$, 陆全明 $\left.{ }^{(1)}\right)^{*}$ ，王水 ${ }^{(1)}$

(1) 中国科学技术大学地球和空间科学学院, 中国科学院基础等离子体物理重点实验室, 合肥 230026;

(2) 空间天气学国家重点实验室, 北京 100190

* 联系人, E-mail: qmlu@ustc.edu.cn

2009-01-19 收稿, 2009-06-16 接受

国家自然科学基金(批准号: 40675008)和中国科学院知识创新工程重要方向项目 (编号: KZCX-YW-206)资助

摘要 在无碰撞磁场重联中, 在分离线的区域的磁压远大于 $\mathrm{X}$ 点附近的磁压, 由此产生 了沿着分离线流向 $\mathrm{X}$ 点的电子束流, 这些电子在 $\mathrm{X}$ 点被加速后, 又沿着靠近分离线内侧 的磁力线流出重联区。一般认为这样的电流体系产生了垂直于重联面的霍尔磁场的四极 型分布, 而且分离线附近区域电子密度会降低. 通过二维粒子模拟方法研究了无引导场

关键词

无碰撞磁场重联 电子密度空穴

四极型分布 时的无碰撞磁场重联, 证实了这样的电流体系. 并且发现四极型磁场的峰值区较分离线 (即电子密度的极小区) 更加靠近电流片内侧, 同时 Cluster 卫星簇的观测资料也证实了这 一现象.

磁场重联提供了一种将磁场能量快速转换成等 离子体动能和热能的物理机制, 它和太阳大气、行星 际空间和地球磁层等空间等离子体中的许多爆发现 象有关 ${ }^{[1,2]}$. 由于这些等离子体中粒子间的碰撞效应 很弱, 可忽略不计, 所以可以认为等离子体是无碰 撞的, 不存在经典意义上的电阻. 最近的研究表明, 霍尔效应在无碰撞等离子体的磁场重联中起着重要 作用, 它使得无碰撞等离子体中磁场能量的快速耗 散成为可能 ${ }^{[3,4]}$.

在无碰撞磁场重联中, 重联区域有多层次结构. 在大于离子惯性尺度 $c / \omega_{p i}$ 的区域起作用的是 Alfven 波, 离子和电子都冻结在磁力线上; 而在小于离子 惯性尺度 $c / \omega_{p i}$ 以内的区域电子和离子的运动是分 离的: 电子被冻结在磁力线上, 而离子则不能被冻 结在磁力线上, 在此区域对重联起主要作用的是哨 声波; 在更小的电子惯性尺度以内, 即使电子的运 动也不能被冻结在磁力线上 ${ }^{[5 \sim 7]}$. 在霍尔磁场重联
中, 在沿着分离线的区域, 由于靠近磁岛两侧的磁 压远大于 $\mathrm{X}$ 点附近的磁压, 产生了沿着分离线流向 $\mathrm{X}$ 点的电子束流, 这些电子在 $\mathrm{X}$ 点被加速后, 又沿着 靠近电流片内侧的磁力线流出重联区. 这样的电流 体系产生了垂直于重联面的霍尔磁场 $B_{y}$ 的四极型分 布 ${ }^{[8,9]}$. 另外观测表明在分离线附近存在着电子的低 密度区域 $[5,10]$. Yang 等人 111$]$ 通过 Hall-MHD 模拟, 表 明在分离线附近区域等离子体密度较低. 在本文中, 我们通过二维粒子模拟程序, 研究了没有初始引导 场时霍尔磁场重联中 $B_{y}$ 的四极型分布和电子密度空 穴的空间位置关系, 并在此基础上和 Cluster 卫星的 观测资料进行了比较.

\section{1 计算模型}

我们采用二维的粒子模拟程序 ${ }^{[12]}$ 来研究无碰撞 磁场重联, 模拟中采用全显格式求解 Maxwell 方程 来更新网格上的电磁场, 带电粒子的运动满足相对 论运动方程. 初始的位形采用 $(x, z)$ 平面内的一维 
Harris 电流片, 其磁场位形由下式给出

$$
\mathbf{B}_{0}(z)=B_{0} \tanh \left[\left(z-\frac{L_{z}}{2}\right) / \delta\right] \mathbf{e}_{x},
$$

其中 $\delta$ 为电流片的半宽度, $L_{z}$ 为 $z$ 方向计算区域的 长度, 对应的粒子数密度为

$$
n(z)=n_{b}+n_{0} \operatorname{sech}^{2}\left[\left(z-\frac{L_{z}}{2}\right) / \delta\right],
$$

其中 $n_{b}$ 为背景等离子体数密度, $n_{0}$ 为 Harris 电流片 密度峰值. 离子和电子的分布函数均为 Maxwell 分 布, 它们在 $y$ 方向的漂移速度满足 $V_{i 0} / V_{e 0}=-T_{i 0} / T_{e 0}$, 其中 $V_{i 0}\left(V_{e 0}\right)$ 和 $T_{i 0}\left(T_{e 0}\right)$ 分别为离子(电子)漂移速度与 初始温度. 模拟中的温度比取 $T_{i 0} / T_{e 0}=5$, 且 $n_{0}=5 n_{b}$. 电流片厚度为 $\delta=0.5 c / \omega_{p i}$, 其中 $c / \omega_{p i}$ 为 由 $n_{0}$ 确定的离子惯性长度. 以前的数值结果表明, 在离子电子质量比足够大时, 离子电子质量比的改 变对重联率没有大的影响 $\frac{[13]}{}$, 在本文中离子电子质 量比取 $m_{i} / m_{e}=100$. 光速取 $c=15 v_{A}$, 其中 $v_{A}$ 为由 $B_{0}$ 与 $n_{0}$ 确定的离子 Alfven 速度, 一般认为光速的改 变对系统的演化不会有大的影响 $\frac{14]}{}$.

我们取 $(x, z)$ 平面上的 $L_{x} \times L_{z}=\left(25.6 c / \omega_{p i}\right) \times$ $\left(12.8 c / \omega_{p i}\right)$ 的区域为计算区域，网格数为 $N_{x} \times N_{z}=512 \times 256$, 空间分辨率为 $\Delta x=\Delta z=0.05 \mathrm{c} /$ $\omega_{p i}=0.5 c / \omega_{p e}$. 时间步长为 $\Omega_{i} \Delta t=0.001$. 模拟中采 用的每种成分的粒子数大约为 $1.0 \times 10^{7} . x$ 方向采用周 期边界条件, 而 $z$ 方向采用反射边界条件. 同时, 为 了使系统尽快进人非线性阶段, 方便我们研究磁场 位形变化比较剧烈的时段, 我们对 Harris 电流片的 位形加人了一个如下的微弱初始磁通扰动

$\psi(x, z)=-\psi_{0} \cos \left[2 \pi\left(x-\frac{L_{x}}{2}\right) / L_{x}\right] \cos \left[2 \pi\left(z-\frac{L_{z}}{2}\right) / L_{z}\right]$.

相应的磁场扰动为

$$
\begin{aligned}
\mathbf{B}= & -2 \pi \psi_{0} / L_{z} \cdot \cos \left[2 \pi\left(x-\frac{L_{x}}{2}\right) / L_{x}\right] \\
& \sin \left[2 \pi\left(z-\frac{L_{z}}{2}\right) / L_{z}\right] \mathbf{e}_{x}+2 \pi \psi_{0} / L_{x} \\
& \sin \left[2 \pi\left(x-\frac{L_{x}}{2}\right) / L_{x}\right] \cos \left[2 \pi\left(z-\frac{L_{z}}{2}\right) / L_{z}\right] \mathbf{e}_{z} .
\end{aligned}
$$

其中 $\psi$ 为磁矢势的 $y$ 分量, $\psi_{0}$ 取 $\psi_{0} /\left(B_{0} c / \omega_{p i}\right)=0.05$; B 为相应的磁场.

\section{2 数值结果}

图 1 给出了磁场重联过程中一组典型时刻下重 联平面内的磁场位形的演化. 这些时刻分别对于重 联即将开始、刚开始、磁岛宽度增长最快和饱和的 时刻. 在重联过程中, 电流片两边初始反向的磁力 线向计算区域的中心相互靠近, 发生断裂后又重新 连接. 重新连接后的磁力线高度扭曲, 在磁张力的 作用下, 磁通量从分离线两侧流出. 随着时间的演 化, 越来越多的磁力线参与了这一重联过程. 在大 约 $\Omega_{i} t=18$ 时, 可发现有明显的 X 点形成, 磁岛半宽 度约为 $1.0 c / \omega_{p i}$. 在 $\Omega_{i} t=23$ 时, 磁岛半宽度约为 $2.0 c / \omega_{p i}$, 此时达到最大重联率. 在大约 $\Omega_{i} t=28$ 时, 重联停止, 磁岛半宽度约为 $2.8 c / \omega_{p i}$.

图 2 所示为重联典型时刻 $\Omega_{i} t=23$ 时各物理量 的分布. 其中图 2(a)和(b)分别为离子与电子在 $(x, z)$ 平面内的速度矢量图, 图 2(c)和(d)分别是垂直于重 联平面的磁场 $B_{y} / B_{0}$ 与电子密度 $\log \left(n / n_{0}\right)$ 的填充等 值线图, 图中背景等值线为 $(x, z)$ 平面内磁场位形, 点虚线为分离线的位置. 从图 2(a)可以看出, 离子 的宏观动力学特征区域只集中在人流区与出流区, 表现出来的特征是重联时离子从电流片两侧向中心 流人, 同时在出流区流出, 其最大出流速度约为 $0.6 v_{A}$. 与之相对应的是, 离子携带着磁通量流向电 流片中心, 在 $\mathrm{X}$ 点附近将磁场能量转化成等离子体 能量, 离子在出流区流出. 由此可以看出, 在磁场 重联过程中, 磁场的宏观拓扑结构的改变几乎取决 于离子的行为. 同时在 $\mathrm{X}$ 点、分离线附近和四极磁 场区域, 没有明显的离子的宏观流动, 而在这些区 域电子的宏观流动却十分明显. 这可以从图 2(b)看 出, 沿着分离线存在明显的流向 $\mathrm{X}$ 点的电子束流, 这是由于在 $X$ 点附近磁场较弱, 电子在磁镜作用下 从强磁场区域沿磁力线向弱磁场区域运动. 另外, 由于在 X点附近存在 $y$ 方向的电场, 电子在 $\mathbf{E} \times \mathbf{B}$ 的 作用下也向 $\mathrm{X}$ 点漂移 ${ }^{[15]}$. 而在分离线内侧大约 $0.2 \sim 0.5 / \omega_{p i}$ 处也存在一从 $\mathrm{X}$ 点流出的电子束流, 这是电子在 $\mathrm{X}$ 点附近加速后沿磁力线流出的结 果 ${ }^{[16]}$, 其最大出流速度为 $2.8 v_{A}$. 这样的电子和离子 的流动图像显然会产生沿着分离线向外的电流, 同 
时在其内侧产生沿磁力线流向 $\mathrm{X}$ 点的电流. 这样的 电流体系就产生了图 2(c) 所示的垂直重联平面的 $B_{y}$ 的四极型分布. 另外由于在分离线附近电子沿着分 离线向 $\mathrm{X}$ 点流动, 在此区域存在着狭长的电子低密 度区域, 这个区域正好对应于图 2(b)中沿着分离线 流向 $\mathrm{X}$ 点的电子束流. 另外在 $\mathrm{X}$ 点的正上和正下方 区域存在指向 $X$ 点的 $\mathbf{E} \times \mathbf{B}$ 的漂移运动, 因而也有
电子的低密度区域.

由上面的分析可知, 沿着分离线流向 $\mathrm{X}$ 点的电 子和在分离线内侧大约 $0.2 \sim 0.5 \mathrm{c} / \omega_{p i}$ 处从 $\mathrm{X}$ 点沿磁 力线流出的电子束流组成的电流体系产生了 $B_{y}$ 的四 极型分布. 因而我们可得出结论 $B_{y}$ 的极大值应该在 分离线的内侧. 图 3 是在沿着 $x /\left(c / \omega_{p i}\right)=7.2$ 这条线(图 $2(\mathrm{c})$ 与 (d)中的虚线)的 $B_{y}$ 和电子密度的剖面. 图中实
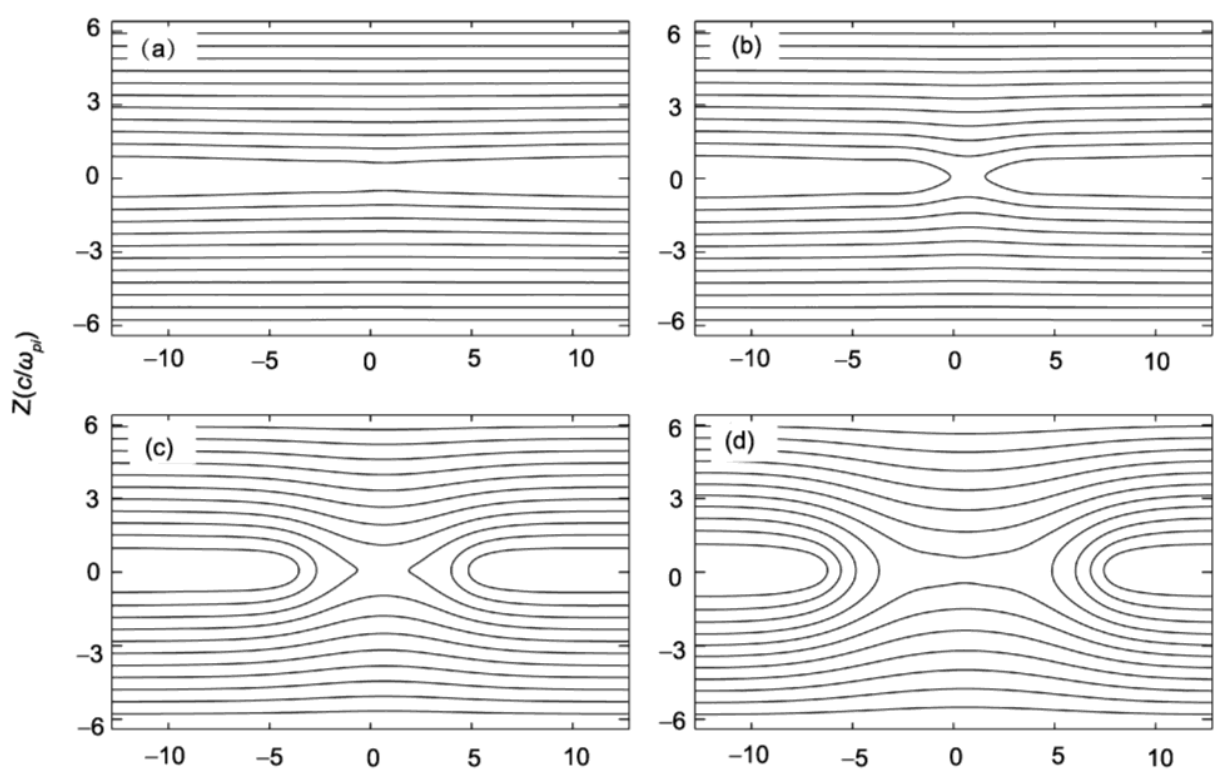

$x /\left(c / \omega_{p i}\right)$

图 1 一组典型时刻下 $(x, z)$ 平面内磁力线位形

(a) $\Omega_{i} t=13$, (b) $\Omega_{i} t=18$, (c) $\Omega_{i} t=23$, (d) $\Omega_{i} t=28$

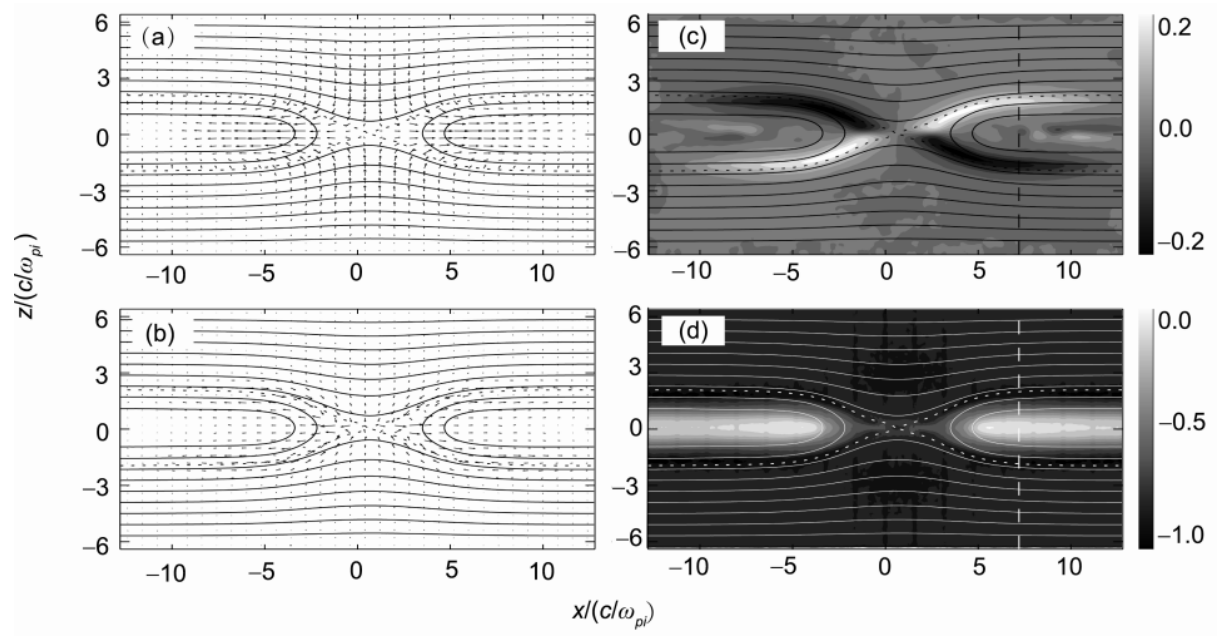

图 $2 \Omega_{i} t=23$ 时刻的各物理量

(a) 离子速度场, (b) 电子速度场, (c) 四极磁场 $B_{y} / B_{0}$, (d) 电子密度 $\log \left(n / n_{0}\right)$ 
线和虚线分别表示 $n / n_{0}$ 和 $B_{y} / B_{0}$ 随 $z$ 的变化, 点虚线 $\mathrm{A}$ 与 $\mathrm{B}$ 分别对应磁场 $B_{y}$ 的峰值与电子密度谷值在 $z$ 方向的位置. 很容易看出四极型磁场 $B_{y}$ 的峰值区更 加靠近电流片内侧, 即在分离线的内侧, 其峰值可通 过 $\mathbf{j}=1 / \mu_{0} \nabla \times \mathbf{B}$ 估算出. 假定是反平行无限长的电流 片, 其产生的磁场强度约为 $B_{y}=\mu_{0} r j$ (其中 $r$ 为两反 平行电流的半间宽, 约为 $0.1 c / \omega_{p i} ; j$ 为两反平行电流 的绝对值之和), 根据公式 $\mathbf{j}=n_{i} e \mathbf{u}_{i}-n_{e} e \mathbf{u}_{e} \approx-n_{e} e \mathbf{u}_{e}$, 可 估算出 $B_{y}$ 的大小约为 $\mu_{0} r n_{e} e u_{e}$, 如取 $n_{e}=n_{0}, u_{e}=2 v_{A}$, 可得 $B_{y}$ 的峰值大小约为 $0.2 B_{0}$.

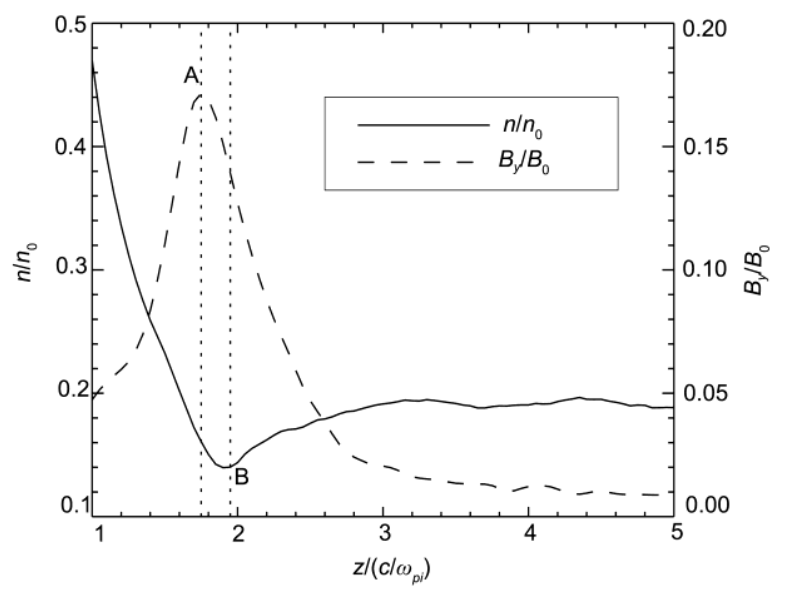

图 $3 \Omega_{i} t=23$ 时刻电子密度与四极磁场在 $x /\left(c / \omega_{p i}\right)=7.2$ 处 的剖面图

\section{3 结论和讨论}

本文通过二维的粒子模拟方法研究了没有初始 引导场时无碰撞磁场重联的结构. 结果表明, 离子决 定了磁场重联的宏观位形, 在沿着分离线的区域, 靠 近磁岛两侧的磁压远大于 $\mathrm{X}$ 点附近的磁压, 这样会 导致沿着分离线产生流向 $\mathrm{X}$ 点的电子束流, 从而使 得分离线附近区域电子密度的降低, 同时在靠近电 流片的内侧又有一支从 $\mathrm{X}$ 点附近加速后流出的电子 束流. 在这样的电流体系的作用下, 产生了垂直于重 联面的霍尔磁场四极型分布. 由此导致的一个结果 是垂直重联平面的四极型磁场的峰值区较分离线(电 子密度的极小区)更加靠近电流片内侧. Hall-MHD 的 模拟结果也表明在等离子体四极型磁场的峰值区较 等离子体密度的极小区更加靠近电流片内侧 $[11]$.
无碰撞磁场重联中, 垂直重联平面的四极磁场 的峰值区对应在密度空洞的极小区的内侧, 这一结 论得到了 Cluster 卫星簇的观测资料的证实. Cluster 卫星是欧空局 2000 年发射的 4 颗相同的卫星组成的 卫星簇. 2001 年 9 月 10 日 07:50 08:05 UT 时间段内, 位于地球磁尾 $19 R_{\mathrm{E}}$ ( $R_{\mathrm{E}}$ 为地球半径)处为 Cluster 卫星 簇探测到一个典型的重联事件. 图 4 显示了这样一个 重联事件的的磁场和等离子体数据图. 图中的数据 由磁通门磁强计 $(\mathrm{FGM})^{[17]}$ 、离子谱分析仪 $(\mathrm{CIS})^{[18]}$ 和 电子电流仪 $(\mathrm{EFW})^{[19]}$ 的测量得到. 磁通门磁强计提供 了磁场三分量数据, 离子光谱分析仪提供了质子高 速整体流数据. 电子电流仪提供卫星电势数据, 再通 过电势计算出电子密度 ${ }^{[201}$. 处理卫星数据时, 均采用 地心太阳磁层 $(\mathrm{GSM})$ 坐标系. 图 4 中 (a) 是电子密度 (此时段内 c3, c4 卫星数据不可用), (b) (d) 分别是磁 场的 3 个分量, (e) 是 $x$ 方向的高速流( $\mathrm{c} 2$ 卫星没有数 据). 电子密度的时间分辨率为 $1 / 5 \mathrm{~s}$, 磁场和高速流 的时间分辨率为 4 s. 07:52 08:02 UT 时间段内, 卫星 观测到高速流由尾侧转变为地侧, 同时 $B_{z}$ 分量由南 侧转变为北侧. 卫星探测到尾向流时, $\mathrm{c} 3$ 卫星位于磁 尾中性片南侧, 观测到的 $B_{y}>0 ; \mathrm{c} 1, \mathrm{c} 2$ 和 $\mathrm{c} 4$ 三颗卫星 位于中性片的北侧, 探测到的 $B_{y}<0$. 当卫星位于高速 地向流中, c3 卫星基本位于磁尾中性片南侧, 观测到 的 $B_{y}<0 ; \mathrm{c} 1, \mathrm{c} 2$ 和 $\mathrm{c} 4$ 三颗卫星基本位于中性片的北侧, 探测到的 $B_{y}>0$. 根据前面的观测事实, 在 07:52 08:02 UT 时间段内, 卫星由尾侧向地侧穿越了 重联 $\mathrm{X}$ 线结构 ${ }^{[21]}$. 穿越过程中, $\mathrm{c} 3$ 卫星基本位于中性 片的南侧, 而其他 3 颗卫星基本位于北侧. 由电子密 度可以看出, 在穿越过程中, $\mathrm{c} 1$ 和 $\mathrm{c} 2$ 卫星多次穿越了 密度空穴(电子密度突然下降约 40\%). 图 5 显示了 07:54:35 07:55:35 UT 时段内 c2 卫星高时间分辨率 $(1 / 22 \mathrm{~s})$ 数据. 图 $5(\mathrm{a})$ 为电子密度数据, (b) (d) 分别为 磁场的 3 个分量数据. 图中实坚线和虚坚线分别表示 $B_{y}$ 峰值和电子密度极小值所在的位置. 由图可见密 度空穴和 Hall 磁场并不重合. 密度极小值出现在 $B_{x}$ 约为 $18 \mathrm{nT}$ 附近, 而 $B_{y}$ 峰值出现在 $B_{x}$ 约为 10 15 nT. 而 $B_{x}$ 值越大意味着卫星位于磁尾中性片越上方, 因 此密度空穴位于 $B_{y}$ 磁场峰值的上方, 即 $B_{y}$ 磁场峰值 更靠近电流片。07:52 08:02 UT 时间段内，卫星多次 穿越了密度空穴, 对其他穿越的分析结果与 07:54:35 07:55:35 UT 时段内 c2 卫星的观测结果一致. 


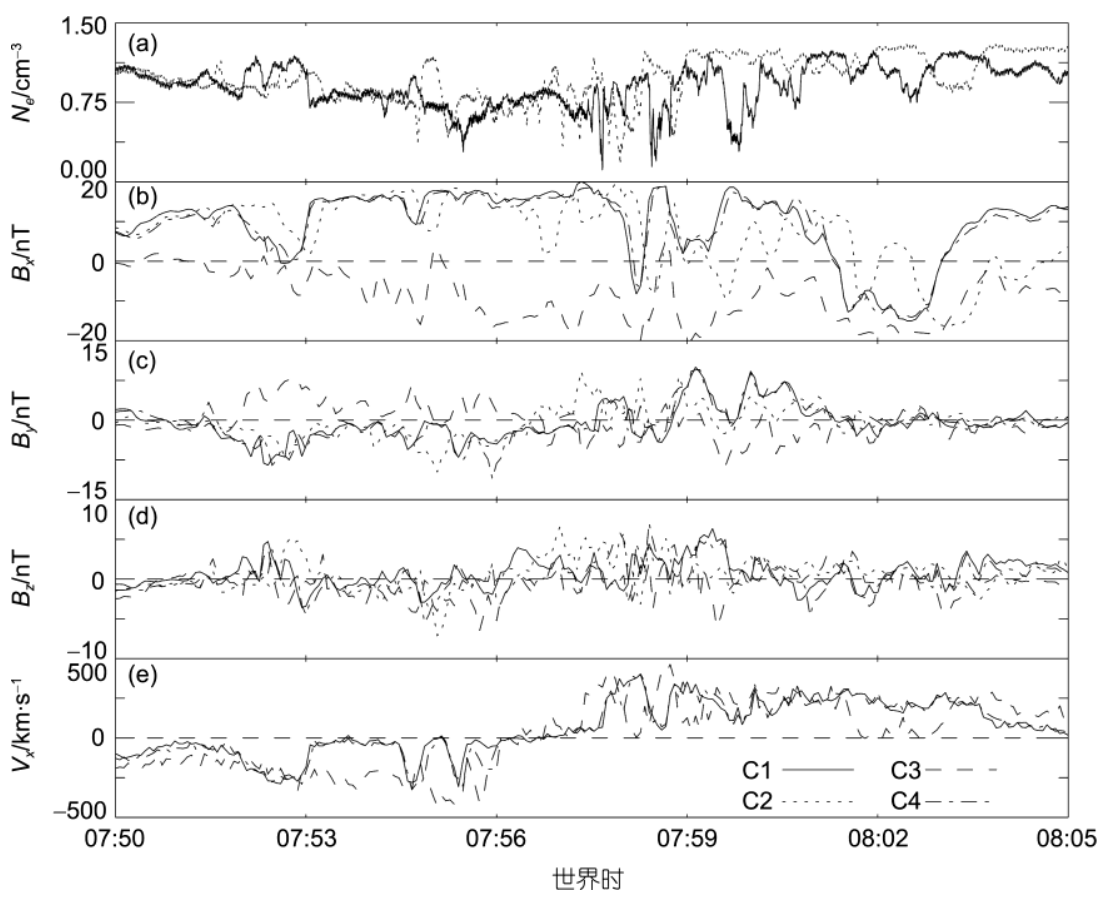

图 4 Cluster 卫星 2001 年9月 10日 07:50 08:05 UT 的观测数据

(a) 电子密度数据; (b) (d)分别表示磁场 $x, y$ 和 $z$ 分量数据; (e) 为 $x$ 方向的高速流数据

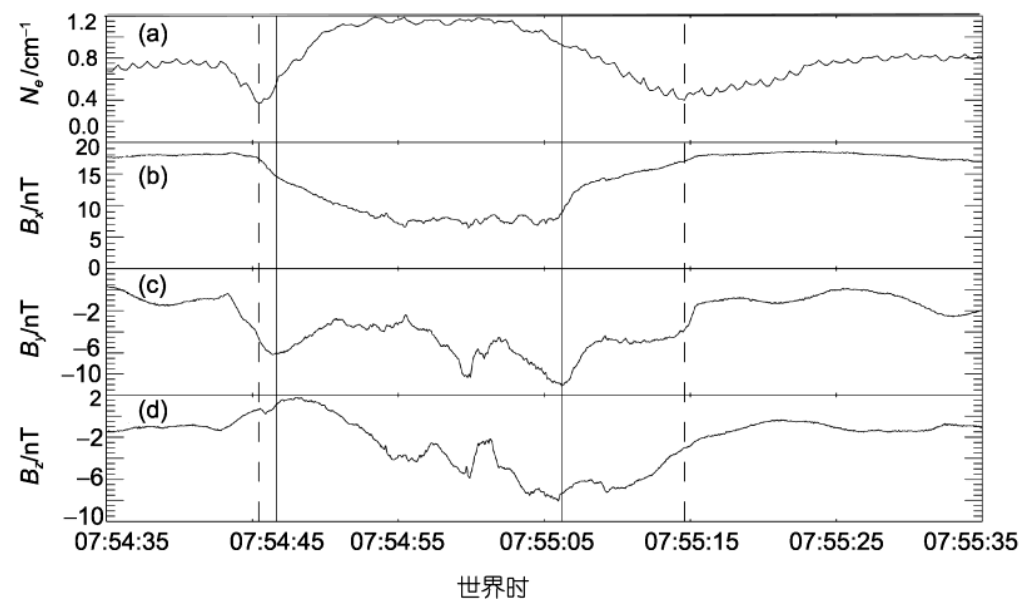

图 5 电子密度和磁场的三分量的 $1 / 22 \mathrm{~s}$ 的数据

实坚线代表 $B_{y}$ 峰值处, 虚坚线表示密度空穴的极小值处

以上的观测结果和我们数值模拟的结果相符, 离线流向 $\mathrm{X}$ 点, 在 $\mathrm{X}$ 点附近加速以后在靠近电流片 也间接证实了电子流动图像的描述, 即电子沿着分 方向的内侧沿磁力线流出.

致谢本文 Cluster卫星数据来源于 ESA Cluster Active Archive. 感谢 FGM, CIS, 和 EFW 仪器组. 


\section{参考文献}

1 Biskamp D. Magnetic Reconnection in Plasma. Cambridge: Cambridge University Press, 2000

Priest E, Forbes T. Magnetic Reconnection: MHD Theory and Applications. Cambridge: Cambridge University Press, 2000

王水, 李罗权. 磁场重联. 合肥: 安徽教育出版社, 1999

Wang X, Bhattacharjee A, Ma Z W. Scaling of collisionless forced reconnection. Phys Rev Lett, 2001, 87: 265003 [doi]

5 Øieroset M, Phan T D, Fujimoto M, et al. In situ detection of collisionless reconnection in the Earth's magnetotail. Nature, 2001, 412: 414-417[doi]

6 Birn J, Drake J F, Shay M A, et al. Geospace environmental modeling (GEM) magnetic reconnection challenge. J Geophys Res, 2001, 106 (A3): 3715-3719[doi]

7 Shay M A, Drake J F, Rogers B N, et al. Alfvénic collisionless reconnection and the Hall term. J Geophys Res, 2001, 106: 3754

8 Pritchett P L. Geospace Environment Modeling magnetic reconnection challenge: Simulations with a full particle electromagnetic code. J Geophys Res, 2001, 108: 3783[doi]

9 Nagai T, Shinohara I, Fujimoto M, et al. Structure of the Hall current system in the vicinity of the magnetic recoonection site. J Geophys Res, 2003, 108: 1357[doi]

10 Mozer F S, Bale S D, Phan T D. Evidence of diffusion regions at a subsolar magnetopause crossing. Phys Rev Lett, 2003, 89: 015002[doi]

11 Yang H A, Jin S P, Zhou G C. Density depletion and Hall effect in magnetic reconnection. J Geophys Res, 2006, 111: A11223[doi]

12 Lu Q M, Cai D S. Implementation of parallel plasma particle-in-cell codes on PC cluster. Comput Phys Commun, 2001, 139: 93-104

13 Guo J, Lu Q M. Effects of ion-electron mass ratio on electron dynamics in collisionless magnetic reconnection. Chin Phys Lett, 2007, 24: 3199 [doi]

14 Birdsall C K, Longdon A B. Plasma Physics via Computer Simulation. New York: McGraw-Hill Book Company, 1991

15 Wang R S, Lu Q M, Huang C, et al. Multispacecraft observation of electron pitch-angle distributions in magnetotail reconnection. J Geophys Res, 2008

16 Fu X R, Lu Q M, Wang S. The process of electron acceleration during collisionless magnetic reconnection. Phys Plasmas, 2006, 13: 012309 [doi]

17 Balogh A, Carr C M, Acuńa M H, et al. The Cluster magnetic field investigation: Overview of in-flight performance and initial results. Ann Geophys, 2001, 19: 1207-1217

18 Gustafsson G, André M, Carozzi T, et al. First results of electric field and density observations by Cluster EFW based on initial months of operation. Ann Geophys, 2001, 19: 1219-1240

19 Pedersen A, Lybekk B, André M, et al. Electron density estimations derived from spacecraft potential measurements on Cluster in tenuous plasma regions. J Geophys Res, 2008, 113: A07S33[doi]

20 Rème H, Aoustin C, Bosqued J M, et al. First multispacecraft ion measurements in and near the Earth's magnetosphere with the identical Cluster ion spectrometry(CIS) experiment. Ann Geophys, 2001, 19: 1303

21 Wang R S, Lu Q M, Guo J, et al. Spatial distribution of energetic electrons during magnetic reconnection. Chin Phys Lett, 2008, 25: 3083-3085[doi] 